\section{ORIGINAL RESEARCH}

\author{
P.C. Sanelli \\ I. Ugorec \\ C.E. Johnson \\ J. Tan \\ A.Z. Segal \\ M. Fink \\ L.A. Heier \\ A.J. Tsiouris \\ J.P. Comunale \\ M. John \\ P.E. Stieg
}

R.D. Zimmerman

A.I. Mushlin

\title{
Using Quantitative CT Perfusion for Evaluation of Delayed Cerebral Ischemia Following Aneurysmal Subarachnoid Hemorrhage
}

BACKGROUND AND PURPOSE: DCI is a serious complication following aneurysmal SAH leading to permanent neurologic deficits, infarction, and death. Our aim was to prospectively evaluate the diagnostic accuracy of CTP and to determine a quantitative threshold for $\mathrm{DCl}$ in aneurysmal SAH.

MATERIALS AND METHODS: Patients with SAH were prospectively enrolled in a protocol approved by the institutional review board. CTP was performed during the typical time period for $\mathrm{DCl}$, between days 6 and 8 following SAH. Quantitative CBF, CBV, and MTT values were obtained by using standard region-of-interest placement sampling of gray matter. The reference standard for $\mathrm{DCl}$ is controversial and consisted of clinical and imaging criteria in this study. In a subanalysis of vasospasm, DSA was used as the reference standard. ROC curves determined the diagnostic accuracy by using AUC. Optimal threshold values were calculated by using the patient population utility method.

RESULTS: Ninety-seven patients were included; $41 \%$ (40/97) had DCl. Overall diagnostic accuracy was $93 \%$ for CBF, $88 \%$ for MTT, and $72 \%$ for CBV. Optimal threshold values were $35 \mathrm{~mL} / 100 \mathrm{~g} / \mathrm{min}(90 \%$ sensitivity, $68 \%$ specificity) for CBF and 5.5 seconds (73\% sensitivity, $79 \%$ specificity) for MTT. In the subanalysis $(n=57), 63 \%$ (36/57) had vasospasm. Overall diagnostic accuracy was $94 \%$ for CBF, $85 \%$ for MTT, and 72\% for CBV. Optimal threshold values were $36.5 \mathrm{~mL} / 100 \mathrm{~g} / \mathrm{min}$ (95\% sensitivity, 70\% specificity) for CBF and 5.4 seconds (78\% sensitivity, 70\% specificity) for MTT.

CONCLUSIONS: CBF and MTT have the highest overall diagnostic accuracy. Threshold values of $35 \mathrm{~mL} / 100 \mathrm{~g} / \mathrm{min}$ for CBF and 5.5-second MTT are suggested for DCl on the basis of the patient population utility method. Absolute threshold values may not be generalizable due to differences in scanner equipment and postprocessing methods.

ABBREVIATIONS: $\mathrm{ACA}=$ anterior cerebral artery; $\mathrm{AIF}=$ arterial input function; $\mathrm{AUC}=$ area under the curve; $\mathrm{Cl}=$ confidence interval; $\mathrm{CTP}=\mathrm{CT}$ perfusion; $\mathrm{DCl}=$ delayed cerebral ischemia; $\mathrm{HHH}=$ hyperdynamic-hypervolemic-hemodilution; NBNCR = net benefit/net cost ratio; N-ICU = neurointensive care unit; $\mathrm{ROC}=$ receiver operating characteristic

A neurysmal SAH is a devastating disease, with patients who survive the initial hemorrhage still at risk for severe complications, such as cerebral ischemia and infarction. Infarction has been detected in up to $81 \%$ of patients. ${ }^{1}$ As many as $46 \%$ of survivors may have long-term cognitive impairment, with an effect on functional status and quality of life. ${ }^{2,3}$ Early diagnosis and prompt medical treatment can prevent these sequelae and improve patient outcomes. ${ }^{4,5}$

\section{Received November 17, 2010; accepted after revision March 22.}

From the Departments of Radiology (P.C.S., C.E.J., L.A.H., A.J.T., J.P.C., M.J., R.D.Z.), Public Health (P.C.S., A.I.M.), and Neurology (A.Z.S., M.F.); Division of Biostatistics (M.J.), Department of Public Health; Department of Neurological Surgery (P.E.S.), New YorkPresbyterian Hospital/Weill Cornell Medical College, New York, New York; Department of Neurology (I.U.), Atlantic Neurosurgical Specialists, Morristown, New Jersey; and Department of Internal Medicine (J.T.), Kettering Medical Center, New York, New York.

This work was supported by grant 5K23NS058387-02 from the NINDS, a component of the $\mathrm{NIH}$.

The views herein are solely the responsibility of the authors and do not necessarily represent the official view of NINDS or NIH.

Please address correspondence to Pina C. Sanelli, MD, MPH, Department of Radiology, New York-Presbyterian Hospital, Weill Cornell Medical College, 525 East 68th St, Starr Pavilion, Starr 630, New York, NY 10065; e-mail: pcs9001@med.cornell.edu

Indicates open access to non-subscribers at www.ajnr.org

Indicates article with supplemental on-line tables

Indicates article with supplemental on-line figures.

http://dx.doi.org/10.3174/ajnr.A2693
The etiology of cerebral ischemia after SAH is complex, and its pathophysiology is poorly understood, often leading to indeterminate diagnoses and delayed treatments. Several terms have emerged to describe its various features, such as "symptomatic vasospasm," "angiographic vasospasm," and "DCI.” DCI has been defined as new focal neurologic impairment or worsening on the Glasgow Coma Scale, after other possible causes of clinical deterioration have been eliminated, and/or new infarction on imaging attributed to vasospasm. ${ }^{6}$ For simplicity, the term vasospasm is reserved for the presence of arterial narrowing on imaging studies. ${ }^{7}$ However, vasospasm and DCI may be related, with arterial narrowing resulting in reduced CBF leading to clinical deterioration and ischemia. Thus, these terms have been considered interchangeable in clinical practice. The caveat is that not all patients with arterial narrowing on angiography develop DCI. Furthermore, not all patients who experience DCI have angiographic vasospasm, which may partly be attributed to circulatory impairment at the microvascular level. ${ }^{8,9}$ Therefore, incorporating both clinical and imaging criteria in the diagnosis of DCI has been reported as most clinically relevant because it has the strongest association with overall poor outcome, cognitive impairment, and reduced quality of life. ${ }^{6}$

Currently, several methods are used to determine DCI, including clinical examination, transcranial Doppler sonogra- 
phy, CTA, and DSA. Some institutions also include CTP to assess the hemodynamic status of the brain to improve the detection of early ischemia. There are several studies in the literature suggesting promising results by using CTP for detection of DCI by identifying perfusion deficits with prolonged MTT and/or decreased CBF. ${ }^{10-13}$ Qualitative perfusion deficits on CTP have shown improved sensitivity and specificity for DCI compared with noncontrast CT and CTA. ${ }^{14}$ Furthermore, Dankbaar et $\mathrm{l}^{12}$ evaluated quantitative CTP and demonstrated significantly lower CBF and higher MTT in patients who developed DCI. However, the most accurate CTP parameter and the optimal threshold for DCI remain controversial. The purpose of this study was to prospectively evaluate the diagnostic accuracy of CTP and to determine a quantitative threshold for DCI in patients with aneurysmal SAH.

\section{Materials and Methods}

\section{Study Population}

A total of 104 patients with aneurysmal SAH were prospectively enrolled in this study from December 2004 to December 2008. Inclusion criteria were adult patients (18 years and older) with documented SAH at admission. SAH was diagnosed by initial noncontrast head CT, CSF analysis, CTA, and/or DSA. Exclusion criteria were a history of iodinated contrast allergy, renal impairment, or pregnancy. The patients and families of all consecutive admissions to the N-ICU with an SAH diagnosis were approached for enrollment. All enrolled subjects underwent surgical clipping and/or endovascular coiling for aneurysm repair, as per the usual standard of care. Institutional review board approval, Health Insurance Portability and Accountability Act compliance, and written informed consent were obtained.

\section{Study Design}

A prospective cohort study was undertaken, including all patients with aneurysmal SAH, to perform CTP on both asymptomatic and symptomatic patients with clinical deterioration. Documentation occurred according to the day following SAH and was performed by the neurologists (I.U., A.Z.S., and M.F., with 8, 12, and 28 years' experience, respectively) in the N-ICU. Day 0 was defined as the day of the $\mathrm{SAH}$ event. Clinical and demographic data collection included age, sex, location of the ruptured aneurysm, type of treatment for aneurysm repair, and the Hunt and Hess grade at presentation. Close observation was maintained to assess deterioration that was not explained by other causes such as aneurysm rebleeding, intracranial hemorrhage, hydrocephalus, infection, metabolic disturbance, seizure, and so forth as determined by clinical assessment, CT, or MR imaging and laboratory studies. Clinical deterioration may manifest with alterations in consciousness, worsening of the Glasgow Coma Scale score, or new neurologic deficits. For patients with limited clinical examinations, particularly patients who were comatose or mechanically ventilated, continuous monitoring of laboratory data and neurologic and systemic parameters was used. DSA with the potential for endovascular treatment was performed in patients with suspected clinical deterioration. We determined the clinical outcome at discharge from the N-ICU, assessing a permanent neurologic deficit on physical examination and/or cerebral infarction on follow-up CT or MR imaging.

\section{CTP Scanning Protocol and Data Processing}

CTP was performed during the typical time period for DCI, between days 6 and 8 in asymptomatic patients and on the day of clinical deterioration in symptomatic patients. Data collection included the day CTP was performed following SAH and CTP's timeframe from the onset of clinical deterioration and its timeframe from when DSA was performed. There is a standard scanning protocol for CTP at our institution by using a LightSpeed or Pro 16 scanner (GE Healthcare, Milwaukee, Wisconsin) with a cine $4 \mathrm{i}$ scanning mode and 45-second acquisition at 1 rotation per second by using $80 \mathrm{kV}$ (peak) and 190 $\mathrm{mA}$. We used a scanning volume of $2.0 \mathrm{~cm}$ consisting of 4 sections at $5.0-\mathrm{mm}$ thickness with inferior extent of the scanning volume selected at the level of the basal ganglia, above the orbits, to minimize radiation exposure to the lenses. Approximately $45 \mathrm{~mL}$ of nonionic iodinated contrast was administered intravenously at $5 \mathrm{~mL} / \mathrm{s}$ by using a power injector with a 5 -second delay.

Postprocessing of the acquired images into CBF, MTT, and CBV maps was performed on an Advantage workstation by using CTP software, Version 3.0 (GE Healthcare). This software uses a deconvolution method, which is considered most accurate for low contrast injection rates. ${ }^{15}$ The postprocessing technique was standardized for all patients according to recommended guidelines ${ }^{16}$ with the AIF as the $\mathrm{A} 2$ segment of the $\mathrm{ACA}^{17}$ and the venous function as the superior sagittal sinus (Fig 1).

The perfusion maps were qualitatively evaluated by 2 neuroradiologists (P.C.S. and A.J.T. with 10 and 7 years' experience, respectively) blinded to clinical and imaging data, to determine the presence of perfusion deficits, defined as areas of decreased $\mathrm{CBF}$ and prolonged MTT based on their radiologic evaluation as performed in clinical practice. Focal perfusion abnormalities due to the primary hemorrhagic event and surgical intervention, as identified on the acquired images from the CTP dataset, were not included as perfusion deficits from DCI. Consensus judgment was determined after reviewing the images independently.

Quantitative analysis was conducted by using a standardized method with contiguous region-of-interest placement, measuring $157 \mathrm{~mm}^{2}$, sampling the cortex and basal ganglia. Each CTP section had up to 28 regions of interest distributed in the following territories: approximately 6 regions of interest in the ACA, 12 regions of interest in the middle cerebral artery, 6 regions of interest in the posterior cerebral artery, and 4 regions of interest in the basal ganglia (Fig 1). CTP studies were analyzed with the reader blinded to all clinical and imaging data to limit test-review bias.

\section{Reference Standard Criteria}

The reference standard for DCI is controversial and continues to undergo review. In this study, a multistage reference standard design was implemented, incorporating the most relevant clinical and imaging outcome measures of DCI, as defined by Reichman et al. ${ }^{18,19}$ On-line Figure 1 demonstrates the flow diagram for assignment of patients in this study.

At the primary level, DSA performed after day 3 was used to determine vasospasm. Angiographic criteria for vasospasm were based on the arterial luminal narrowing compared with the normal parent vessel and comparison with DSA performed on admission. Mild angiographic vasospasm was $<50 \%$ arterial narrowing; moderate vasospasm was $50 \%-75 \%$ narrowing; and severe vasospasm was $>75 \%$ narrowing. Interpretation of DSA was performed by 2 observers, an interventional neuroradiologist who performed the examination (with either 10 or 25 years' experience) and a neuroradiologist blinded to all patient information (C.E.J. with 22 years' experience). For disagree- 

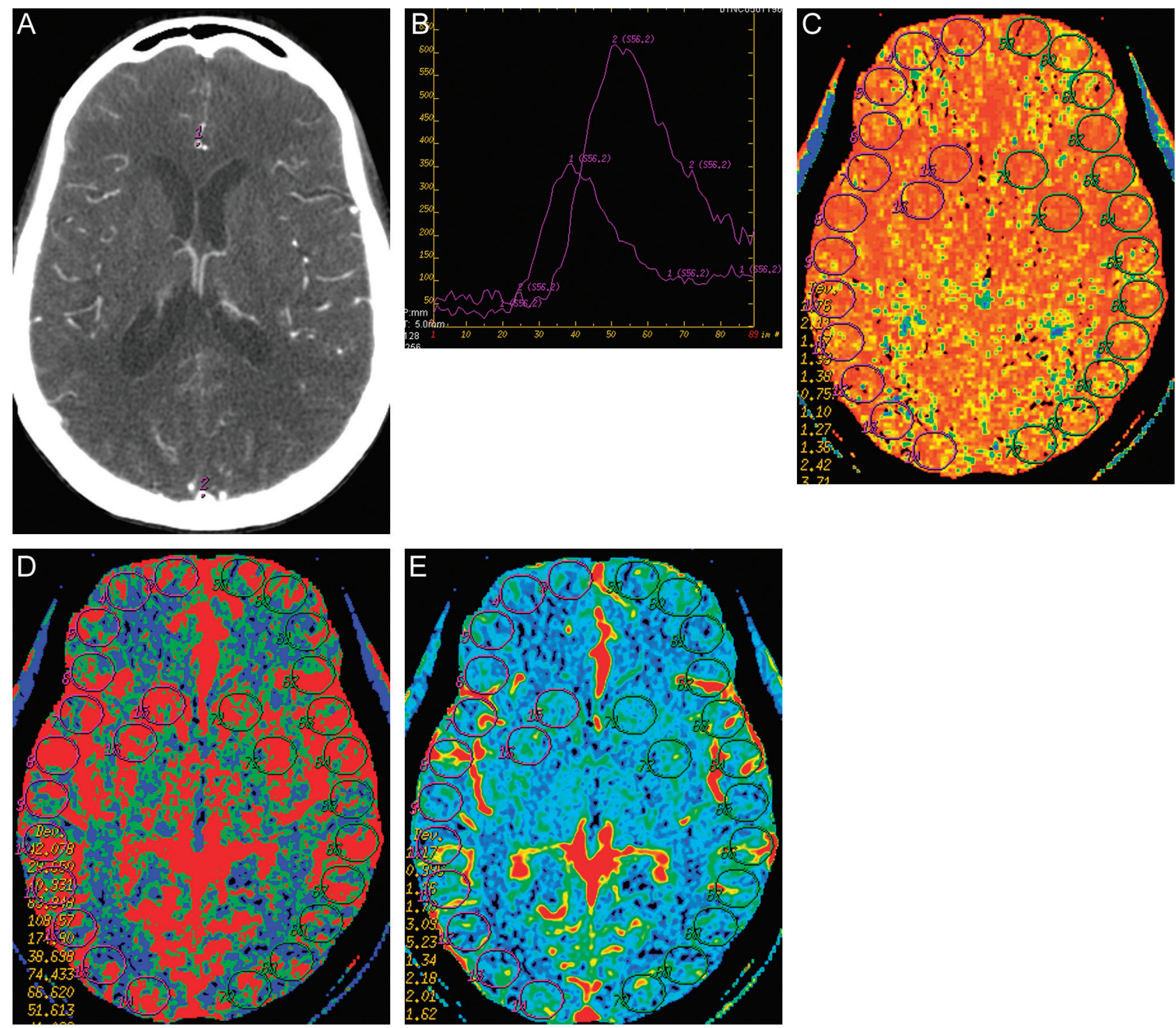

Fig 1. The CTP postprocessing technique was standardized for all patients by using a deconvolution method. $A$, Standardized selection of the AIF (1) as the A2 segment of the anterior cerebral artery and venous function (2) as the superior sagittal sinus. B, The arterial (1) and venous (2) time-attenuation curves are used to select the pre-enhancement and postenhancement cutoff values according to recommended guidelines. $C-E$, Quantitative analysis was conducted by using a standardized method of contiguous region-of-interest placement, measuring $157 \mathrm{~mm}^{2}$, sampling the cortex and basal ganglia for the MTT, CBF, and CBV maps, respectively.

ments, a third neuroradiologist (P.C.S. with 10 years' experience) independently reviewed the examination in a blinded fashion.

In those patients who did not undergo DSA, clinical or imaging criteria or both for the outcome events of DCI were used as the reference standard at the secondary level. On discharge from the N-ICU, the presence of either of the following criteria supported the diagnosis of DCI:

1) Clinical criteria: permanent neurologic deficit on clinical examination, distinct from the deficit at baseline produced by the SAH event or surgical intervention, which was not attributable to other causes.

2) Imaging criteria: cerebral infarction on follow-up CT and/or MR imaging that occurred after day 4 . This criterion has been used to effectively exclude primary brain damage from $\mathrm{SAH}$ and/or surgical intervention. ${ }^{6,20}$

For patients who did not meet the above criteria and did not receive $\mathrm{HHH}$ therapy, a "no DCI" diagnosis was assigned. However, patients without the above criteria who received $\mathrm{HHH}$ therapy proceeded to the tertiary level for assessment of a response to treatment. Patients who demonstrated an improvement in clinical deterioration following $\mathrm{HHH}$ therapy were considered responders to appropriate treatment, and the response supported the diagnosis of DCI. Patients who did not improve following treatment and were found to have another cause for their symptoms were assigned as having no evidence of DCI. However, patients who did not have another cause for their symptoms underwent further testing with DSA and were then diagnosed at the primary level.

\section{Statistical Analysis}

Only CTP examinations performed at the diagnostic stage, before treatment for DCI and before cerebral infarction, were included in the analysis to minimize confounding bias because both treatment and infarction affect cerebral perfusion and the diagnosis. The mean CBF, $\mathrm{CBV}$, and MTT values were calculated for the DCI and no DCI groups. For patients with focal perfusion deficits, regions of interest within the affected region were isolated and arithmetic means were calculated. In patients without focal deficits, all regions of interest for all 4 section locations were included in the arithmetic means. To minimize the contribu- 


\begin{tabular}{|c|c|c|c|c|c|c|}
\hline \multicolumn{7}{|c|}{ Clinical and demographic characteristics of the study population and subgroup } \\
\hline & \multicolumn{3}{|c|}{ Study Population $(n=97)$} & \multicolumn{3}{|c|}{ Subgroup $^{\mathrm{a}}(n=57)$} \\
\hline & $\begin{array}{c}\text { All } \\
(n=97)\end{array}$ & $\begin{array}{c}\mathrm{DCl} \\
(n=40)\end{array}$ & $\begin{array}{l}\text { No DCl } \\
(n=57)\end{array}$ & $\begin{array}{c}\text { All } \\
(n=57)\end{array}$ & $\begin{array}{l}\text { Vasospasm } \\
(n=36)\end{array}$ & $\begin{array}{c}\text { No Vasospasm } \\
(n=21)\end{array}$ \\
\hline Age (yr) (median) & 49 & 48 & 49 & 51 & 48 & 55 \\
\hline Range & $28-80$ & $30-78$ & $28-80$ & $28-80$ & $30-78$ & $28-80$ \\
\hline \multicolumn{7}{|l|}{ Sex } \\
\hline Male (\%) (No.) & $27(26 / 97)$ & $25(10 / 40)$ & $28(16 / 57)$ & $25(14 / 57)$ & $25(9 / 36)$ & $24(5 / 21)$ \\
\hline Female (\%) (№.) & $73(71 / 97)$ & $75(30 / 40)$ & $72(41 / 57)$ & $75(43 / 57)$ & $75(27 / 36)$ & $76(16 / 21)$ \\
\hline \multicolumn{7}{|l|}{ Aneurysm location } \\
\hline Anterior & $92(89 / 97)$ & $90(36 / 40)$ & $93(53 / 57)$ & $95(54 / 57)$ & $92(33 / 36)$ & $100(21 / 21)$ \\
\hline Posterior & $8(8 / 97)$ & $10(4 / 40)$ & $7(4 / 57)$ & $5(3 / 57)$ & $8(3 / 36)$ & $0(0 / 21)$ \\
\hline \multicolumn{7}{|l|}{ Treatment type } \\
\hline Surgical clipping & $55(53 / 97)$ & $50(20 / 40)$ & $58(33 / 57)$ & $65(37 / 57)$ & $56(20 / 36)$ & $81(17 / 21)$ \\
\hline Coil embolization & $45(44 / 97)$ & $50(20 / 40)$ & $42(24 / 57)$ & $35(20 / 57)$ & $44(16 / 36)$ & $19(4 / 21)$ \\
\hline \multicolumn{7}{|l|}{ Hunt and Hess grade } \\
\hline Low (grades 1 and 2) & $53(51 / 97)$ & $35(14 / 40)$ & $63(36 / 57)$ & $46(26 / 57)$ & $36(13 / 36)$ & $62(13 / 21)$ \\
\hline High (grades 3, 4, and 5) & $47(46 / 97)$ & $65(26 / 40)$ & $37(21 / 57)$ & $54(31 / 57)$ & $64(23 / 36)$ & $38(8 / 21)$ \\
\hline Clinical deterioration & $45(44 / 97)$ & $83(33 / 40)$ & $19(11 / 57)$ & $67(38 / 57)$ & $81(29 / 36)$ & $43(9 / 21)$ \\
\hline
\end{tabular}

tion of vascular pixels from large vessels, we excluded the CBF values of $>100 \mathrm{~mL} / 100 \mathrm{~g} / \mathrm{min}$ from the statistical analysis and did not use these in calculating the mean CBF, CBV, and MTT values since this method for the evaluation of ischemia has been published. ${ }^{21}$ The regions of interest in the perfusion abnormalities due to the primary hemorrhagic event and/or surgical intervention, as identified on the acquired images from the CTP dataset, were not included in the statistical analysis. Normality of the distribution of continuous variables was assessed by plotting histograms and the Shapiro-Wilk test. Comparison between the groups was performed by using a 2-tailed $t$ test for normal variables and the Wilcoxon rank sum test for non-normal variables. Statistical significance was accepted at $P<.05$. The $95 \% \mathrm{CI}$ was used as the measure of variance. ROC curves were generated for each CTP parameter, and the AUC was calculated to quantify overall diagnostic accuracy. We fitted maximumlikelihood ROC models and 95\% CIs, assuming a binormal distribution for the underlying latent variable. The threshold value was assessed by using the patient population utility method, which incorporates the NBNCR for the SAH population. (Please refer to the On-line Appendix for detailed description of this method.) The statistical analysis was performed by a biostatistician (M.J. with 5 years' experience) by using STATA, Version 10 (StataCorp, College Station, Texas) and R, Version 2.7.2 (http://cran.r-project.org/bin/windows/base/old/2.7.2/) software.

Analysis was also conducted with the same methods mentioned above in a subgroup by using DSA as the reference standard to specifically evaluate the diagnostic accuracy of CTP for vasospasm.

\section{Results}

\section{Study Population Characteristics}

Patient recruitment occurred from December 2004 to December 2008. Online Figure 2 is the flow diagram for patient enrollment. Ninety-seven patients were included in the statistical analysis. Forty-one percent (40/97) of patients had DCI and 59\% (57/97) did not, as determined by the reference standard. On-line Figure 1 demonstrates the assignment of patients by the reference standard for each level. In the subgroup $(n=57), 63 \%(36 / 57)$ of patients had vasospasm and $37 \%$ (21/57) did not, as determined by DSA.

Clinical and demographic characteristics of the study population and subgroup are presented in the Table. The imaging characteristics of the study population and subgroup are shown in On-line Table 1. CTP was performed during the typical time period expected for DCI and on the same day that symptoms occurred in all groups.

\section{Clinical Outcomes}

No adverse events from CTP were reported in the study population. In the DCI group, 48\% (19/40) of patients had no permanent neurologic deficit, 40\% (16/40) had permanent neurologic deficits, and 13\% (5/40) died during hospitalization. In the no DCI group, $88 \%(50 / 57)$ of patients had no permanent neurologic deficit, 12\% (7/57) had permanent neurologic deficits referable to causes other than DCI (eg, postsurgical contusion and/or ischemia), and none died during hospitalization.

\section{Quantitative CTP Analysis}

In the study population, no CTP results were excluded from the analysis, including potential outliers. The mean quantitative values of the CTP parameters and 95\% CIs for the study population and subgroup are presented in On-line Table 2. There are statistically significant differences in the CBF, CBV, and MTT values for the 2 groups. Figure 2 demonstrates the ROC curves and AUC for each CTP parameter with its 95\% CI for the study population and subgroup, respectively. CBF has the highest overall diagnostic accuracy followed by MTT and CBV. The optimal operating point on these ROC curves was determined by using the patient population utility method, which incorporates the pretest probability of disease, the expected net benefit of correct diagnoses (true-positives), and the expected net harms associated with false-positive results (On-line Appendix). According to this method, the CBF threshold was $35 \mathrm{~mL} / 100 \mathrm{~g} / \mathrm{min}$ (90\% sensitivity, $68 \%$ specificity) and the MTT threshold was 5.5 seconds (73\% sensitivity, $79 \%$ specificity) for the diagnosis of DCI. In the subgroup of patients with DSA as the reference standard for vasospasm, the CBF threshold was $36.5 \mathrm{~mL} / 100 \mathrm{~g} / \mathrm{min}$ (95\% sensitivity, $70 \%$ specificity) and MTT threshold was 5.4 seconds (78\% sensitivity, $70 \%$ specificity). 


\section{Discussion}

Cerebral ischemia following SAH is a complex entity involving delayed narrowing of the intracranial arteries that may lead to clinical deterioration, infarction, and death. A review of the literature reveals that inconsistent terminology has been used to describe this entity in clinical trials and observational studies. The relationship between vasospasm seen on angiography and clinical deterioration and ischemia from DCI has led to imaging studies often being used as surrogate diagnostic tools. ${ }^{7}$ Given the variable association among these terms, our study focused on evaluating CTP as it would be used in clinical practice to prospectively identify patients with DCI, as defined by both clinical and imaging criteria.

Several publications suggest that CTP is a promising tool in detecting DCI. Patients with regional hypoperfusion deficits more often experience DCI and are at risk for infarction. ${ }^{14,22,23}$ Our study supports these findings, with qualitative CTP deficits seen in $95 \%$ of patients with DCI compared with $18 \%$ without DCI. More recently, investigations have focused on the diagnostic accuracy of quantitative CTP for DCI and determining a diagnostic threshold. Dankbaar et $\mathrm{al}^{12}$ reported that MTT can detect patients with DCI by using an absolute MTT threshold of 5.9 seconds. On the other hand, several studies support the finding that CBF has the highest sensitivity and specificity, ${ }^{24}$ concluding that patients with $\mathrm{CBF}$ reduction on CTP, ${ }^{10}$ xenon-enhanced $\mathrm{CT},{ }^{25}$ or position-emission tomography ${ }^{26}$ imaging were more vulnerable to developing DCI. Our study confirms CBF and MTT as the most useful CTP parameters for diagnosing DCI. Our large prospective cohort should be highly generalizable to clinical practice because it consists of both asymptomatic and symptomatic patients, as well as patients who did or did not undergo DSA (On-line Appendix). Furthermore, our results indicate that CBF has the best discrimination ability for DCI (Fig 2), with the highest overall diagnostic accuracy (0.93) compared with MTT (0.88) and CBV (0.72). However, the 95\% CIs of CBF and MTT are overlapping, and thereby a statistically significant difference is not achieved between these 2 parameters. The best operating point on these ROC curves was determined as $35 \mathrm{~mL} / 100 \mathrm{~g} / \mathrm{min}$ ( $90 \%$ sensitivity, $68 \%$ specificity) for CBF and 5.5 seconds (73\% sensitivity, 79\% specificity) for MTT calculated by the patient population utility method, which incorporates key factors, such as the net benefit/harm ratio and the prevalence of DCI. Therefore, this statistical method minimizes both false-negative and false-positive errors to maximize the average state of health of the SAH population.

Other studies in the literature have evaluated CTP for the diagnosis of vasospasm by using DSA as the reference standard. High sensitivity and specificity have been reported for qualitative CTP deficits in the detection of vasospasm and the prediction of secondary infarction. ${ }^{10,13,23,27,28}$ Our subgroup analysis supports these findings with qualitative CTP deficits seen in $97 \%$ of patients with vasospasm compared with $24 \%$ without vasospasm. More specifically, several studies focused on assessing the degree of arterial narrowing and the presence of a perfusion abnormality in its corresponding territory. Aralasmak et $\mathrm{al}^{13}$ reported that a perfusion abnormality was noted in $83 \%$ of patients with severe vasospasm compared with $26 \%$ with mild-moderate vasospasm and $15 \%$ without vasospasm. Dankbaar et $\mathrm{al}^{22}$ demonstrated that the flow terri- tory of the vessel with the most severe vasospasm corresponded with the least perfused region on CTP.

Furthermore, Wintermark et $\mathrm{al}^{11}$ evaluated quantitative CTP for detection of vasospasm in a retrospective study and reported that MTT had the highest diagnostic accuracy (91.6\%) by using a threshold of 6.4 seconds, closely followed by $\mathrm{CBF}(91.4 \%)$ with a threshold of $44.3 \mathrm{~mL} / 100 \mathrm{~g} / \mathrm{min}$. Our subgroup analysis also revealed that CBF and MTT had the highest diagnostic accuracy for vasospasm on DSA. With the patient population utility method, a CBF threshold of $36.5 \mathrm{~mL} / 100 \mathrm{~g} / \mathrm{min}$ (95\% sensitivity, 70\% specificity) and an MTT threshold of 5.4 seconds (78\% sensitivity, $70 \%$ specificity) were calculated. Overall, the CTP findings in the study population to determine DCI and in the subgroup to determine vasospasm demonstrate similar results. These results support the association of arterial narrowing and ischemia in $\mathrm{SAH}$ and promote the added value of using DSA in the diagnosis of DCI to guide patient management.

Currently, the definitions of DCI and vasospasm are undergoing review by expert panels and investigators to improve uniformity in research studies evaluating outcomes in patients with SAH. Vergouwen et $\mathrm{al}^{7}$ recently provided an expert opinion as to the use of the following outcome measures for DCI: 1) cerebral infarction identified on $\mathrm{CT}$ or MR imaging after exclusion of procedure-related infarctions, and 2) functionalbased outcome measures. The term "vasospasm" is recommended for patients with arterial narrowing on imaging studies. ${ }^{7}$ DSA is considered the reference standard for vasospasm, given its superior spatial resolution. However, as CT scanner technology continues to improve, other noninvasive methods such as CTA may also be used to detect vasospasm, as supported by a recent meta-analysis.

In clinical practice, angiographic evidence of vasospasm remains as a surrogate diagnostic tool to prospectively determine diagnosis and treatment of cerebral ischemia after $\mathrm{SAH}$, particularly given its association with perfusion deficits. Therefore, a previously published and validated reference standard that includes these relevant outcome measures was used in this study. Reichman et al ${ }^{18,19}$ thoroughly describe the implementation of this reference standard along with its strengths and limitations. Several strengths of this reference standard include its widespread applicability to the entire $\mathrm{SAH}$ population, incorporation of the most relevant outcome measures of cerebral ischemia after $\mathrm{SAH}$, thorough use of criteria to exclude other causes of clinical deterioration and cerebral infarction, and its consideration of treatment effects in determining diagnosis. Incorporating a reference standard based on a clinical practice approach, by using intensive clinical monitoring combined with serial imaging examinations to prospectively identify patients with DCI may assist in bridging the gap between clinical research and clinical practice.

We acknowledge several limitations in this study, including limited brain coverage for evaluation of perfusion deficits provided by CTP. However, emerging CT scanner technology will provide broader coverage in the future, and these data may be applicable to this improvement. Another limitation is that the region of CTP imaging was not coordinated with the location of the arterial narrowing on DSA or the region referable to symptoms. Therefore, it is conceivable that this region may not have been imaged on CTP or may possibly have been averaged with a 

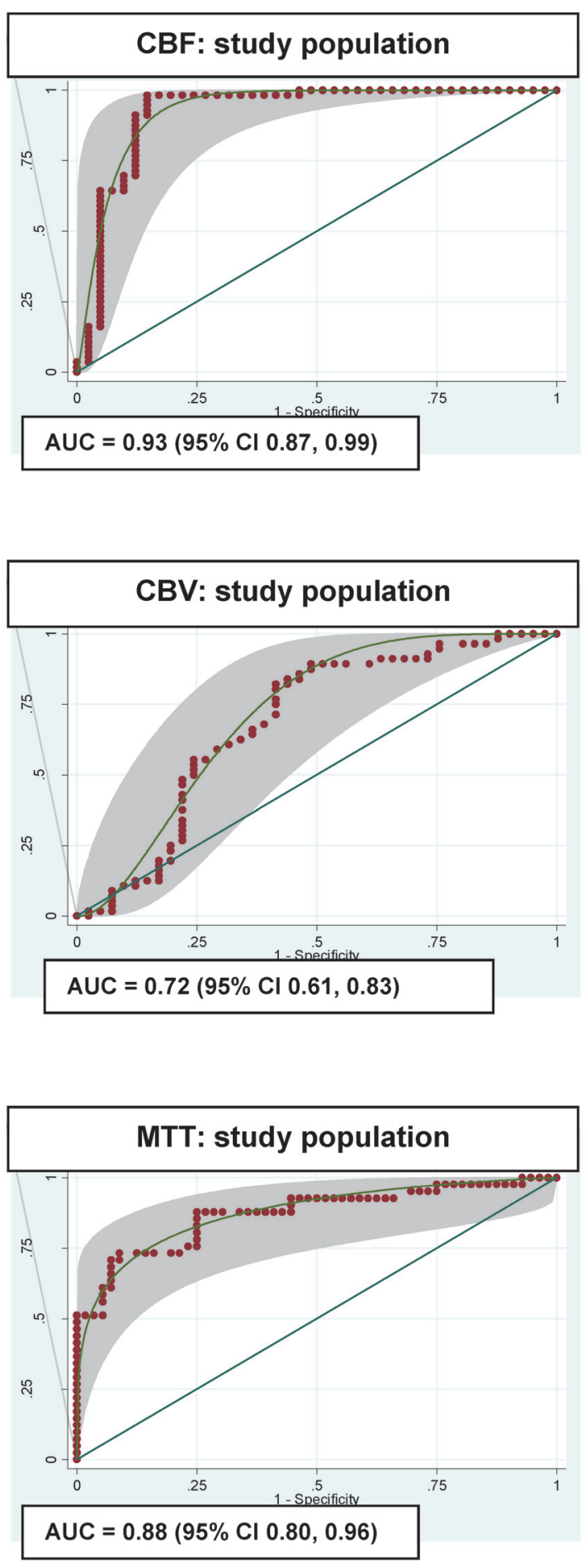

Fig 2. ROC curves for the study population $(n=97)$ and subgroup $(n=57)$.

larger region of normal perfusion, resulting in increased falsenegatives and lower diagnostic accuracy in our study. This method was not used to reduce work-up and observer bias because we did not have knowledge of the clinical examination and DSA results before CTP scanning and interpretation.

Overall, there is an inherent limitation in applying quantitative CTP findings in clinical practice. Caution is urged when using absolute quantitative CTP values for the diagnosis and management of patients. Often, these threshold values are
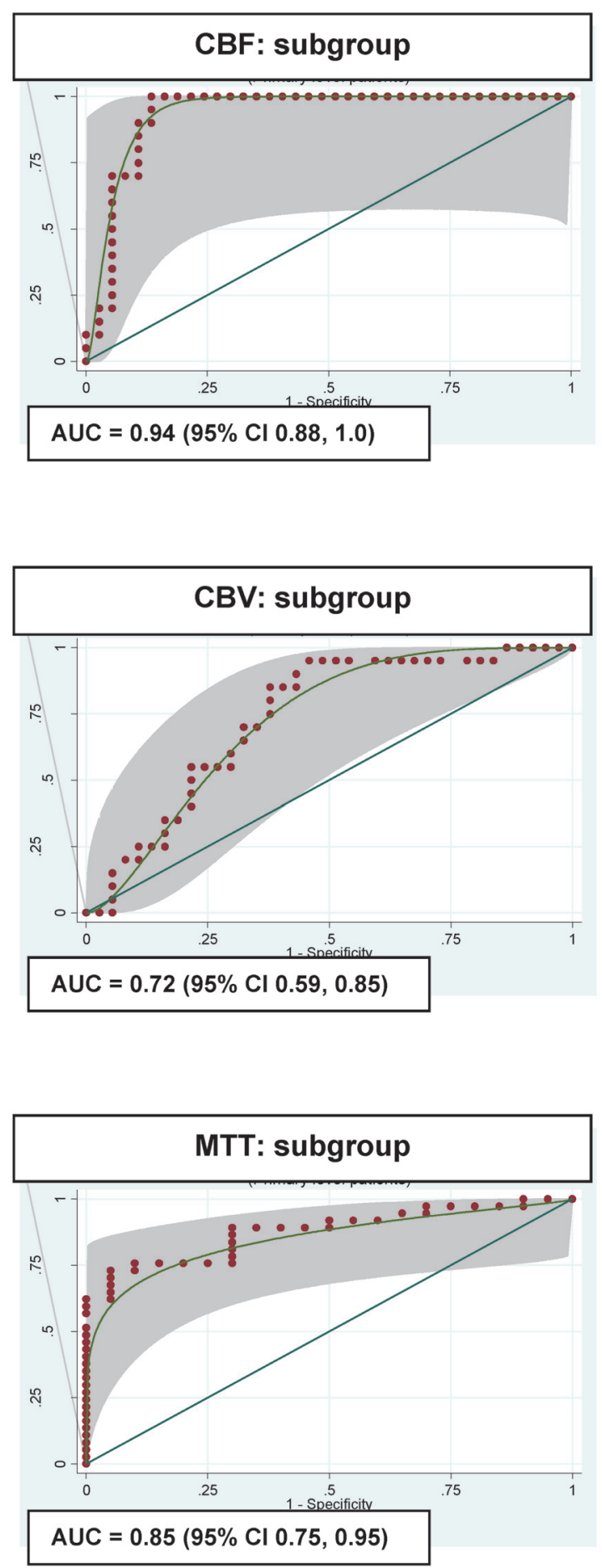

only valid for the constellation of the type of hardware equipment and software program used in a specific study. Careful interpretation of absolute values is suggested because the quantitation of the perfusion parameters relies on the assumption that the selected arterial input and venous outflow functions represent the true inflow and outflow measures for the tissue bed being analyzed. Selection of the AIF in patients with acute cerebral ischemia remains controversial, and there is no consensus to date. However, several studies support the find- 
ing that there is no significant variation in the quantitative CTP parameters of patients with acute cerebral ischemia when using different AIF locations. ${ }^{16,17,30-32}$

Current expert opinion favors the use of relative rather than absolute CTP values, given the potential for variability in absolute quantitation of CTP parameters and the dependence of these values on an appropriate but often arbitrary venous output scaling factor. ${ }^{33}$ Dankbaar et $\mathrm{al}^{12}$ have demonstrated that overall relative threshold values had better diagnostic properties than absolute measurements, possibly because relative measurements reduce the variability caused by the postprocessing steps. ${ }^{34}$ However, absolute threshold values were used in our study because cerebral ischemia after SAH may occur bilaterally and diffusely in the brain; therefore, standardized methods are difficult to implement in this patient population. Most important, standardization and validation of CTP methodology and postprocessing techniques are necessary for its widespread implementation in patients with SAH.

\section{Conclusions}

CTP adds important physiologic data to the clinical information and anatomic imaging of patients with SAH. CBF and MTT emerged as the CTP parameters with the greatest discrimination ability for DCI. A CBF threshold of $35 \mathrm{~mL} / 100 \mathrm{~g} / \mathrm{min}$ and an MTT threshold of 5.5 seconds were calculated by using the patient population utility method, which statistically infers the maximum average state of health for this population. However, caution is recommended in using specific absolute threshold values for CTP because the differences in scanner equipment and postprocessing methods may affect its generalizability. Therefore, future steps are needed to assess the performance of these threshold values in improving clinical outcomes by using a large prospective clinical trial study design.

Disclosures: Matthew Fink, Consultant: Novartis, Details: consultant regarding new pharmaceutical uses, Other Financial Interests: Editor of Neurology ALERT, Details: AHC Media LLC. Apostolos John Tsiouris, Consultant. BioClinica, Details: BioClinica is an imaging core lab for which I consult on the design of phase II research studies that use imaging to assess drug efficacy. Compensation from consultation is $<\$ 5000 /$ year. I occasionally also act as a blinded expert reader or adjudicator for phase II studies. Alvin I. Mushlin, Consultant. GE Healthcare, Details: Member of the advisory board for the GE Radiology Research Academic Fellowship program.

\section{References}

1. Kivisaari RP, Salonen O, Servo A, et al. MR imaging after aneurysmal subarachnoid hemorrhage and surgery: a long-term follow-up study. AJNR Am J Neuroradiol 2001;22:1143-48

2. Mayer SA, Kreiter KT, Copeland D, et al. Global and domain-specific cognitive impairment and outcome after subarachnoid hemorrhage. Neurology 2002; 59:1750-58

3. Hackett ML, Anderson CS. Health outcomes 1 year after subarachnoid hemorrhage: an international population-based study. Neurology 2000;55: $658-62$

4. Solomon RA, Fink ME, Lennihan L. Early aneurysm surgery and prophylactic hypervolemic hypertensive therapy for the treatment of aneurysmal subarachnoid hemorrhage. Neurosurgery 1988;23:699-704

5. Morgan MK, Jonker B, Finfer S, et al. Aggressive management of aneurysmal subarachnoid hemorrhage based on a papaverine angioplasty protocol. $\mathrm{J} \mathrm{Clin}$ Neurosci 2000;7:305-08

6. Frontera JA, Fernandez A, Schmidt JM, et al. Defining vasospasm after subarachnoid hemorrhage: what is the most clinically relevant definition? Stroke 2009;40:1963-68

7. Vergouwen MD, Vermeulen M, van Gijn J, et al. Definition of delayed cerebral ischemia after aneurysmal subarachnoid hemorrhage as an outcome event in clinical trials and observational studies: proposal of a multidisciplinary research group. Stroke 2010;41:2391-95

8. Vergouwen MD, Vermeulen M, Coert BA, et al. Microthrombosis after aneu- rysmal subarachnoid hemorrhage: an additional explanation for delayed cerebral ischemia. J Cereb Blood Flow Metab 2008;28:1761-70

9. Ohkuma H, Manabe $\mathrm{H}$, Tanaka $\mathrm{M}$, et al. Impact of cerebral microcirculatory changes on cerebral blood flow during cerebral vasospasm after aneurysmal subarachnoid hemorrhage. Stroke 2000;31:1621-27

10. Nabavi DG, LeBlanc LM, Baxter B, et al. Monitoring cerebral perfusion after subarachnoid hemorrhage using CT. Neuroradiology 2001;43:7-16

11. Wintermark M, Ko NU, Smith WS, et al. Vasospasm after subarachnoid hemorrhage: utility of perfusion CT and CT angiography on diagnosis and management. AJNR Am J Neuroradiol 2006;27:26-34

12. Dankbaar JW, de Rooij NK, Rijsdijk M, et al. Diagnostic threshold values of cerebral perfusion measured with computed tomography for delayed cerebral ischemia after aneurysmal subarachnoid hemorrhage. Stroke 2010;41: 1927-32

13. Aralasmak A, Akyuz M, Ozkaynak C, et al. CT angiography and perfusion imaging in patients with subarachnoid hemorrhage: correlation of vasospasm to perfusion abnormality. Neuroradiology 2009;51:85-93

14. Dankbaar JW, de Rooij NK, Velthius BK, et al. Diagnosing delayed cerebral ischemia with different CT modalities in patients with subarachnoid hemorrhage with clinical deterioration. Stroke 2009;40:3493-98

15. Wintermark M, Maeder P, Thiran JP, et al. Quantitative assessment of regional blood flows by perfusion CT studies at low injection rates: a critical review of the underlying theoretical models. Eur Radiol 2001;11:1220-30

16. Sanelli PC, Lev MH, Eastwood JD, et al. The effect of varying user-selected input parameters on quantitative values in CT perfusion maps. Acad Radiol 2004;11:1085-92

17. Wintermark M, Lau BC, Chen J, et al. The anterior cerebral artery is an appropriate arterial input function for perfusion-CT processing in patients with acute stroke. Neuroradiology 2008;50:227-36

18. Reichman MB, Greenberg ED, Gold RL, et al. Developing patient-centered outcome measures for evaluating vasospasm in aneurysmal subarachnoid hemorrhage. Academic Radiology 2009;16:541-45

19. Reichman M, Gold RL, Greenberg E, et al. Validation of a new reference standard for the diagnosis of vasospasm. Acad Radiol 2010;17:1083-89

20. Powsner RA, O’Tuama LA, Jabre A, et al. SPECT imaging in cerebral vasospasm following subarachnoid hemorrhage. J Nucl Med 1997;39:765-69

21. Murphy BD, Fox AJ, Lee DH, et al. Identification of penumbra and infarct in acute ischemic stroke using computed tomography perfusion-derived blood flow and blood volume measurements. Stroke 2006;37:1771-77

22. Dankbaar JW, Rijsdijk M, van der Schaaf IC, et al. Relationship between vasospasm, cerebral perfusion, and delayed cerebral ischemia after aneurysmal subarachnoid hemorrhage. Neuroradiology 2009;51:813-19

23. Pham M, Johnson A, Bartsch AJ, et al. CT perfusion predicts secondary cerebral infarction after aneurismal subarachnoid hemorrhage. Neurology 2007;69:762-65

24. van der Schaaf I, Wermer MJ, van der Graaf Y, et al. CT after subarachnoid hemorrhage: relation of cerebral perfusion to delayed cerebral ischemia. $\mathrm{Neu}$ rology 2006;66:1533-38

25. Knuckey NW, Fox RA, Surveyor I, et al. Early cerebral blood flow and computerized tomography in predicting ischemia after cerebral aneurysm rupture. J Neurosurg 1985;62:850-55

26. Frykholm P, Andersson JL, Langstrom B, et al. Haemodynamic and metabolic disturbances in the acute stage of subarachnoid haemorrhage demonstrated by PET. Acta Neurol Scand 2004;109:25-32

27. Binaghi S, Colleoni ML, Maeder $\mathrm{P}$, et al. CT angiography and perfusion CT in cerebral vasospasm after subarachnoid hemorrhage. AJNR Am J Neuroradiol 2007;28:750-58

28. Wintermark M, Dillon WP, Smith WS, et al. Visual grading system for vasospasm based on perfusion CT imaging: comparisons with conventional angiography and quantitative perfusion CT. Cerebrovasc Dis 2008;26:163-70

29. Greenberg ED, Gold R, Reichman M, et al. Diagnostic accuracy of CT angiography and CT perfusion for cerebral vasospasm: a meta-analysis. AJNR Am J Neuroradiol 2010;31:1853-60

30. Ferreira RM, Lev MH, Goldmakher GV, et al. Arterial input function placement for accurate CT perfusion map construction in acute stroke. AJR Am J Roentgenol 2010;194:1330-36

31. Sasaki M, Kudo K, Ogasawara K, et al. Tracer delay-insensitive algorithm can improve reliability of CT perfusion imaging for cerebrovascular stenoocclusive disease: comparison with quantitative single-photon emission CT. AJNR Am J Neuroradiol 2009;30:188-93

32. Wittsack HJ, Wohlschlager AM, Ritzl EK, et al. CT-perfusion imaging of the human brain: advanced deconvolution analysis using circulant singular value decomposition. Comput Med Imaging Graph 2008;32:67-77

33. Konstas AA, Goldmakher GV, Lee T-Y, et al. Theoretic basis and technical implementations of CT perfusion in acute ischemic stroke. Part 2. Technical implementations. AJNR Am J Neuroradiol 2009;30:885-92

34. Kealey SM, Loving VA, Delong DM, et al. User-defined vascular input function curves: influence on mean perfusion parameter values and signal-to-noise ratio. Radiology 2004;231:587-93 